Algebraic 85 Geometric Topology

Volume 2 (2002) 285-296

Published: 18 April 2002

ATG

\title{
All flat manifolds are cusps of hyperbolic orbifolds
}

\author{
D.D. LONG
}

A.W. REID

\begin{abstract}
We show that all closed flat $n$-manifolds are diffeomorphic to a cusp crosssection in a finite volume hyperbolic $n+1$-orbifold.
\end{abstract}

AMS Classification 57M50; 57R99

Keywords Flat manifolds, hyperbolic orbifold, cusp cross-sections

\section{Introduction}

By a flat $n$-manifold (resp. flat $n$-orbifold) we mean a manifold (resp. orbifold) $\mathbf{E}^{n} / \Gamma$ where $\mathbf{E}^{n}$ is Euclidean n-space and $\Gamma$ a discrete, cocompact torsion-free subgroup of $\operatorname{Isom}\left(\mathbf{E}^{n}\right)$ (resp. $\Gamma$ has elements of finite order). In [7], Hamrick and Royster resolved a longstanding conjecture by showing that every flat $n$-manifold bounds an $(n+1)$-dimensional manifold, in the sense that each diffeomorphism class has a representative that bounds.

Flat manifolds and orbifolds are connected with hyperbolic orbifolds via the structure of the cusp ends of finite volume hyperbolic orbifolds - if $M^{n+1}$ is a non-compact finite volume hyperbolic orbifold, then by a standard analysis of the thin parts, a cusp cross-section is a flat $n$-orbifold (see below and [14]). In the early eighties, motivated by this and work of Gromov [6], Farrell and Zdravkovska [5] posed the following geometric version:

Question Does the diffeomorphism class of every flat $n$-manifold have a representative $W$ which arises as the cusp cross-section of a finite volume 1-cusped hyperbolic $(n+1)$-manifold?

We note that it makes sense to ask only for bounding up to diffeomorphism type, since it is a well-known consequence of Mostow Rigidity that there are algebraic restrictions on the isometry type of the flat $n$-manifolds that can arise as cusp cross sections of finite volume hyperbolic $(n+1)$-manifolds. 
In this generality, the question has a positive answer in dimension 2 , that is, for the torus and Klein bottle, but as is shown in [10] is false in dimension 3 (and indeed in all dimensions $4 k-1 \geq 3$ ). On the other hand, since finite volume, non-compact hyperbolic manifolds exist in all dimensions (see $\S 6$ for example), this together with the fact that maximal abelian subgroups are separable (see below and [9]) can be used to deduce the $n$-torus is a cusp cross section of a hyperbolic manifold with possibly many cusps.

In this paper we shall show:

Theorem 1.1 For every $n \geq 2$, the diffeomorphism class of every flat $n$ manifold has a representative $W$ which arises as some cusp cross-section of a finite volume cusped hyperbolic $(n+1)$-orbifold.

As already remarked, much more is known in dimension 2, and we only include this since the argument also works there. In dimension 3, [11] proves a little more, namely that every flat 3-manifold is a cusp cross-section of a hyperbolic 4-manifold, but even there, the number of cusps is not known to be one. Also in dimension 3, in [13], it is shown by an ad hoc argument that of the 10 diffeomorphism types of flat 3-manifolds, 7 have representatives that arise as a cusp cross-section in some 5 cusped hyperbolic manifold related to gluings of the ideal 24-cell in $\mathbf{H}^{4}$.

The proof of Theorem 1.1 uses arithmetic methods, together with some extra control one can arrange to make a separability argument. The main ingredient in the proof is the following (see $\S 2$ for definitions and notation):

Theorem 1.2 Let $\Gamma$ be the fundamental group of a flat $n$-manifold. Then there is a quadratic form $q_{n+2}$ defined over $\mathbf{Q}$, of signature $(n+1,1)$ for which $\Gamma$ embeds as a subgroup of $\mathrm{O}_{0}\left(q_{n+2} ; \mathbf{Z}\right)$.

The group $\mathrm{O}_{0}\left(q_{n+2} ; \mathbf{Z}\right)$ is arithmetic, and hence of finite co-volume acting on $\mathbf{H}^{n+1}$. The proof of Theorem 1.1 is then completed by using a subgroup separability argument to pass to a subgroup of finite index in $\mathrm{O}_{0}\left(q_{n+2} ; \mathbf{Z}\right)$ for which the group $\Gamma$ is a maximal peripheral subgroup.

As with [11], we cannot guarantee 1-cusped examples, and at present, we have been unable to pass to manifolds, even assuming separability of geometrically finite subgroups.

It appears that the question in its full generality is much harder to approach, for example, it appears be unknown, whether, for $n \geq 4$, there even exist 1 -cusped hyperbolic $n$-manifolds of finite volume. As far as the authors are aware, this is known only in dimensions 2 and 3. 


\section{Preliminaries.}

\subsection{Quadratic forms}

We need to recall some standard facts about quadratic forms and orthogonal groups of such forms; [8] is a standard reference.

If $f$ is a quadratic form in $n+1$ variables with coefficients in $K$ and associated symmetric matrix $F$, let

$$
\mathrm{O}(f)=\left\{X \in \mathrm{GL}(n+1, \mathbf{C}) \mid X^{t} F X=F\right\}
$$

be the Orthogonal group of $f$, and

$$
\mathrm{SO}(f)=\mathrm{O}(f) \cap \mathrm{SL}(n+1, \mathbf{C}),
$$

the Special Orthogonal group of $f$. These are algebraic groups defined over $K$. If $L$ is a subring of $\mathbf{C}$ we denote the set of $L$-points of these groups by $\mathrm{O}(f ; L)$ (resp. $\mathrm{SO}(f ; L))$.

Definition. Two $n$-dimensional quadratic forms $f$ and $q$ defined over a field $K$ (with associated symmetric matrices $F$ and $Q$ ) are equivalent over $K$ if there exists $P \in \operatorname{GL}(n, K)$ with $P^{t} F P=Q$.

If $K \subset \mathbf{R}$ is a number field, and $R_{K}$ its ring of integers, then $\mathrm{SO}\left(f ; R_{K}\right)$ is an arithmetic subgroup of $\mathrm{SO}(f ; \mathbf{R}),[3]$ or [2]. In particular $\mathrm{SO}\left(f ; R_{K}\right)$ has finite co-volume acting on an associated symmetric space. The following is well-known and proved in [1] for example.

Lemma 2.1 If $f$ and $q$ are equivalent over $K$ then:

- $\mathrm{SO}(f ; \mathbf{R})$ is conjugate to $\mathrm{SO}(q ; \mathbf{R})$ and $\mathrm{SO}(f ; K)$ is conjugate to $\mathrm{SO}(q ; K)$.

- $\mathrm{SO}\left(f ; R_{K}\right)$ is conjugate to a subgroup of $\mathrm{SO}(q ; K)$ commensurable with $\mathrm{SO}\left(q ; R_{K}\right)$.

\subsection{Crystallographic groups and hyperbolic orbifolds}

We record some facts about crystallographic and Bieberbach groups that we will need, see [4] for a comprehensive discussion of these groups, and [14] Theorem 4.2.2 for the theorem stated below.

An $n$-dimensional crystallographic group is a cocompact discrete group of isometries of $\mathbf{E}^{n}$. When $\Gamma$ is torsion-free it is called a Bieberbach group. By 
Bieberbach's Third Theorem (see [4]), the number of n-dimensional crystallographic groups up to affine equivalence is finite. What we require is summarized in the following from [14] page 222:

Theorem 2.2 An n-dimensional crystallographic group $\Gamma$ contains a normal subgroup of finite index consisting of translations, that is free abelian of rank $n$. The maximal such subgroup is characterized as the unique maximal abelian subgroup of finite index in $\Gamma$.

Theorem 2.2 implies that associated to an $n$-dimensional crystallographic group $\Gamma$ is a finite group $\theta(\Gamma)$, the holonomy group, and an extension:

$$
1 \longrightarrow \mathbf{Z}^{n} \longrightarrow \Gamma \longrightarrow \theta(\Gamma) \longrightarrow 1 \text {. }
$$

When $\Gamma$ is a Bieberbach group we get a free action on $\mathbf{E}^{n}$, by rigid motions, that is for all $\gamma \in \Gamma$, and $v \in \mathbf{E}^{n}$,

$$
v \rightarrow \theta(\gamma) v+t_{\gamma}
$$

for some $t_{\gamma} \in \mathbf{E}^{n}$ (see [4] for details).

\subsection{1}

We refer the reader to [12] or [14] for further details on what is contained in the next two subsections.

Equip $\mathbf{R}^{n+1}$ with the quadratic form $f_{n}=\langle-1,1 \ldots, 1,1\rangle$ of signature $(n, 1)$. The connected component of the identity in $\mathrm{O}\left(f_{n} ; \mathbf{R}\right)$ will be denoted $\mathrm{O}_{0}\left(f_{n} ; \mathbf{R}\right)$. This group preserves the upper sheet of the hyperboloid $f_{n}(x)=-1$ but contains reflections so reverses orientation. We identify $\mathrm{O}_{0}\left(f_{n} ; \mathbf{R}\right)$ with $\operatorname{Isom}\left(\mathbf{H}^{n}\right)$. Passing to the connected component of the identity in $\operatorname{SO}\left(f_{n} ; \mathbf{R}\right)$, denoted $\mathrm{SO}_{0}\left(f_{n} ; \mathbf{R}\right)$ (which has index 4 in $\mathrm{O}\left(f_{n} ; \mathbf{R}\right)$ ), gives a group which may be identified with $\operatorname{Isom}_{+}\left(\mathbf{H}^{n}\right)$; it preserves the upper sheet of the hyperboloid $f_{n}(x)=-1$ and the orientation. Given a (discrete) subgroup $\Delta$ of $\mathrm{O}(n, 1 ; \mathbf{R})$, $\Delta \cap \mathrm{SO}_{0}(n, 1 ; \mathbf{R})$ has index $\leq 4$ in $\Gamma$.

\subsection{2}

An element of $\mathrm{O}_{0}\left(f_{n} ; \mathbf{R}\right)$ is parabolic (resp. elliptic) if it has a unique fixed point which lies on $S_{\infty}^{n-1}$ (resp. has a fixed point in $\mathbf{H}^{n}$ ). Given a non-compact hyperbolic $n$-orbifold $Q=\mathbf{H}^{n} / \Delta$ of finite volume, and $C$ a cusp cross-section 
of $Q$, then there is a subgroup $\Delta_{C}<\Delta$ consisting of parabolic and elliptic elements having an invariant horosphere $\mathcal{H}$ such that $\mathcal{H} / \Delta_{C}=C$. The group $\Delta_{C}$ is a crystallographic group. This group is called a maximal peripheral subgroup of $\Delta$.

In terms of the model above, an element is parabolic if and only if it is not elliptic and leaves invariant a unique light-like vector $v$. Furthermore, in the context of $\Delta$ and $\Delta_{C}$ above, all elements of $\Delta_{C}$ will preserve this unique lightlike vector. We summarize what we need.

Lemma 2.3 Let $Q=\mathbf{H}^{n+1} / \Delta$ be a non-compact finite volume hyperbolic orbifold. $\Delta_{C}$ is a maximal peripheral subgroup of $\Delta$ if and only if $\Delta_{C}$ is the maximal subgroup of $\Delta$ leaving the light-like vector $v$ invariant. Furthermore when $\Delta_{C}$ is maximal, by choice of a horosphere $\mathcal{H}, \mathcal{H} / \Delta_{C} \cong C$ is an embedded cusp cross-section of $Q$.

\section{2 .3}

We record the following for convenience concerning arithmetic subgroups of $\operatorname{Isom}\left(\mathbf{H}^{n}\right)$. For more details, see [3], [2] and [16].

Let $f$ be a diagonal quadratic form with rational coefficients and signature $(n, 1)$. Thus there is a $P \in \mathrm{GL}(n+1, \mathbf{R})$ such that $P^{t} F P=F_{n}$, and so the group $P \mathrm{O}_{0}(f ; \mathbf{Z}) P^{-1}$ defines a discrete arithmetic subgroup of $\operatorname{Isom}\left(\mathbf{H}^{n}\right)$. The theory of arithmetic groups then gives,

Theorem 2.4 In the notation above, the groups $P_{0}(f ; \mathbf{Z}) P^{-1}$ are finite covolume subgroups of $\operatorname{Isom}\left(\mathbf{H}^{n}\right)$.

In what follows, we will suppress the conjugation by $P$. A group $\mathrm{O}_{0}(f ; \mathbf{Z})$ (and hence the conjugate in $\operatorname{Isom}\left(\mathbf{H}^{n}\right)$ ) is cocompact if and only if the form $f$ does not represent 0 non-trivially with values in $\mathbf{Q}$, see [3]. Whenever $n \geq 4$, the arithmetic groups constructed above are non-cocompact, since it is well known every indefinite quadratic form over $\mathbf{Q}$ in at least 5 variables represents 0 nontrivially, see [8]. In fact up to commensurability, all non-cocompact arithmetic subgroups of $\mathrm{O}_{0}\left(f_{n} ; \mathbf{R}\right)$ arise from this construction (see [16]).

\subsection{Some technical lemmas}

In this section we gather together a collection of well-known results on separability properties of groups that will be used to pass from Theorem 1.2 to Theorem 1.1. 
Recall that a subgroup $H$ of a group $G$ is separable in $G$ if, given any $g \in G \backslash H$, there exists a subgroup $K<G$ of finite index with $H<K$ and $g \notin K$. $G$ is called subgroup separable (or $L E R F$ ) if all finitely generated subgroups of $G$ are separable in $G$. The profinite topology on a group $G$ is defined by proclaiming all finite index subgroups of $G$ to be a basis of open neighbourhoods of the identity. Since open subgroups are closed in the profinite topology, the following reformulates separability:

Lemma 2.5 Let $G$ be a group and $H<G$ is a subgroup. $G$ is $H$-subgroup separable if and only if $H$ is closed in the profinite topology on $G$.

Lemma 2.6 Let $G$ be a group and $H<K<G$. Assume that $H$ is separable in $G$ and that $[K: H]<\infty$. Then $K$ is separable in $G$.

Proof By Lemma 2.5, $H$ is closed in the profinite topology on $G$. Standard properties of topological groups imply that any coset $g H$ of $H$ in $G$ is therefore a closed subset. Since $[K: H]<\infty, K$ is a finite union of closed sets, hence closed, and therefore separable in $G$.

The following is also well-known (see [9]):

Lemma 2.7 Let $G$ be a residually finite group, and $A$ a maximal abelian subgroup. Then $A$ is separable in $G$.

\section{Proof of Theorems 1.1 and 1.2}

This section is devoted to proving theorems 1.1 and 1.2. We prove the latter first, which we restate for the reader's convenience:

Theorem 1.2 Let $\Gamma$ be the fundamental group of a flat $n$-manifold. Then there is a quadratic form $q_{n+2}$ defined over $\mathbf{Q}$, of signature $(n+1,1)$ for which $\Gamma$ embeds as a subgroup of $\mathrm{O}_{0}\left(q_{n+2} ; \mathbf{Z}\right)$.

Before embarking on the proof we remark that the first part of the proof can be replaced by the argument in the proof of Bieberbach's third theorem giving an integral representation into $\mathrm{GL}(n+1, \mathbf{Z})$ of a Bieberbach group. However, we will use some additional features of the construction given below in completing the proof of Theorem 1.1. 
Proof of Theorem 1.2 Suppose that $\Gamma$ is the fundamental group of a flat $n$-manifold, so as discussed in $\S 2.2$, we have a free action of $\Gamma$ on $\mathbf{E}^{n}$ by rigid motions. Thus, if $g \in \Gamma$, then $g$ acts as

$$
v \rightarrow \theta(g) v+t_{g}
$$

and the assignment

$$
g \rightarrow \theta(g)
$$

is a homomorphism of $\Gamma$ to its holonomy group $\theta(\Gamma)$, with kernel the maximal translation subgroup of $\Gamma$.

Suppose that $\mu_{1}, \ldots, \mu_{n}$ generate the maximal normal free abelian $\mathbf{Z}^{n}$ in $G$, where $\mu_{i}$ acts as translation by $\mathbf{m}_{i}$, where we declare that this is the vector $\mathbf{m}_{i}=(0, \ldots, 1, \ldots, 0)$, one in $i-t h$ place. The group $\Gamma$ acts by conjugacy on the subgroup $\left\langle\mu_{1}, \ldots, \mu_{n}\right\rangle$ and a calculation reveals that $g \mu_{i} g^{-1}$ is the translation given by

$$
v \rightarrow v+\theta(g) \mathbf{m}_{i} .
$$

The normality of the translation subgroup shows that

$$
g \mu_{i} g^{-1}=\mu_{1}^{a_{i, 1}(g)} \ldots \ldots \mu_{n}^{a_{i, k}(g)}
$$

for some collection of integers $\left\{a_{i, j}(g)\right\}$. Equating these two statements gives a finite integral representation of $G$ given by

$$
\theta(g) \mathbf{m}_{i}=\Sigma_{j} a_{i, j}(g) \mathbf{m}_{j}
$$

We now construct an integral linear representation of $\Gamma$, as follows. Choose a presentation for the group $\Gamma$ using generators $g_{1}, \ldots . ., g_{p}$ and with relations $w_{t}\left(g_{1}, \ldots, g_{p}\right)=I$, and add all relators which say $w_{j}\left(g_{1}, \ldots, g_{p}\right)=\mu_{j}$ for each $1 \leq j \leq n$. (These ensure that the chosen $\mathbf{m}_{i}$ 's don't change.) Each $g_{i}$ acts as

$$
v \rightarrow \theta\left(g_{i}\right) v+t_{i},
$$

so that expanding the equations coming from the relators, we get a collection of equations for the $t_{i}$ 's with rational coefficients which have some solution (for example, that coming from the identity representation that we are given for $\Gamma$ as a Bieberbach group).

It follows that there are rational solutions to these equations and we claim that any such solution gives a faithful and rational representation of $G$. Pick any rational solution and regard this as a representation $\rho: \Gamma \rightarrow \rho(\Gamma)$. The conditions imposed by the second batch of equations guarantee that the restriction of $\rho$ to the translation subgroup of $\Gamma$ is actually the identity homomorphism. Since the translation subgroup is isomorphic to $\mathbf{Z}^{n}$ and this is Hopfian, it follows 
that $\operatorname{ker}(\rho)$ avoids the translation subgroup of finite index and hence $\operatorname{ker}(\rho)$ is trivial, since $\Gamma$ is torsion free. This shows that $\rho$ is an isomorphism as required. It follows that there is a rational solution for the $t_{i}$ 's in terms of the $\mathbf{m}_{i}$ 's. Convert the affine representation on $\mathbf{E}^{n}$ to a rational linear representation on $\mathbf{E}^{n+1}$ by

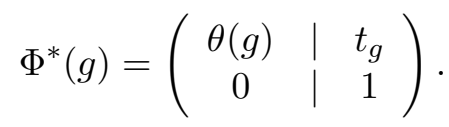

This is a faithful rational linear representation of $\Gamma$, which comes from coning the given action of $\Gamma$ by rigid motions in the hyperplane $e_{n+1}=1$ to the origin in $\mathbf{E}^{n+1}$. By conjugating the representation, we may rescale the vector $e_{n+1}$ and thus arrange that the representation $\Phi^{*}$ is actually by integral matrices.

It is slightly more convenient at this stage to define a new faithful integral representation by setting

$$
\Phi(g)=\left(\Phi^{*}(g)^{T}\right)^{-1}=\left(\begin{array}{c|c}
\theta\left(g^{-1}\right)^{T} & 0 \\
-\theta\left(g^{-1}\right)\left(t_{g}\right)^{T} & 1
\end{array}\right)
$$

where $A^{T}$ denotes transpose. We can then extend this representation to $\mathbf{E}^{n+2}$ by mapping $g$ to

$$
\hat{\Phi}(g)=\left(\begin{array}{c|c}
\Phi(g) & \mathbf{v}_{g} \\
0 & 1
\end{array}\right)
$$

where the column vector $\mathbf{v}_{g}$ is to be determined.

Now let $\langle$,$\rangle be any \theta^{T}$ invariant positive definite inner product on the $\mathbf{Z}$-module $\left\langle\mu_{1}, \ldots, \mu_{n}\right\rangle$; such an inner product exists by taking a random inner product and forming the $\theta$-average. Let $D$ be the symmetric rational matrix associated to this form in the $\left\{\mathbf{m}_{i}\right\}$ basis. Extend this form to $\mathbf{E}^{n+2}$ by summing on a subspace $H_{2}$, which in the language of quadratic forms is a hyperbolic plane. More precisely, we let $H_{2}$ denote the 2-dimensional form $2 X Y$, with associated symmetric matrix $\left(\begin{array}{ll}0 & 1 \\ 1 & 0\end{array}\right)$ (see [8] Chapter 1). The form $D \oplus H_{2}$ now has signature $(n+1,1)$.

Denoting vectors lying in $\mathbf{E}^{n}$ by $w$ and the last two dimensions by $v_{1}$ and $v_{2}$, it is a simple matter of linear algebra to show that $\mathbf{v}_{g}=\left(W_{g}, \tau_{g}\right) \in \mathbf{E}^{n} \oplus\left\langle v_{1}\right\rangle$ may be chosen so that each $\hat{\Phi}(g)$ is an isometry of the form $D \oplus H_{2}$.

The linear algebra suggests that the matrices $\hat{\Phi}(g)$ may be nonintegral in the last column, since the initial solution vectors $\mathbf{v}_{g}$ need only be rational. However, conjugating by a matrix of the form

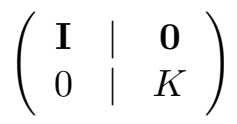

Algebraic 83 Geometric Topology, Volume 2 (2002) 
we may find a new collection of matrices which are the same save in the last column, and which has $\mathbf{v}_{g}$ replaced by $K . \mathbf{v}_{g}$. In particular, for suitable $K$ we may arrange that the conjugated representation is integral.

After this conjugation, the new representation now leaves invariant a different form, but this new form is rationally equivalent to $D \oplus \mathrm{H}_{2}$; in particular, it continues to have signature $(n+1,1)$.

Claim With this choice, we get a faithful integral representation of the group $\Gamma$.

Proof We need only show that the relations in $\Gamma$ hold. Faithfulness will follow, because if a product of these matrices is the identity, then it must at least be the identity in the $n+1$ representation which is already a faithful representation of $\Gamma$.

We prove the claim by showing that any isometry, $\gamma$ say, which is the identity on the upper left $n+1 \times n+1$ block is in fact the identity.

Note that by construction, every $\gamma \in \Gamma$ stabilizes $v_{1}$.

Pick a random $w \in \mathbf{E}^{n}$. Then $0=\left\langle w, v_{2}\right\rangle=\left\langle\gamma w, \gamma v_{2}\right\rangle=\left\langle w, w^{\prime}+\xi v_{1}+v_{2}\right\rangle=$ $\left\langle w, w^{\prime}\right\rangle$. This holds for all $w$, so that $w^{\prime}=0$.

Also $0=\left\langle v_{2}, v_{2}\right\rangle=\left\langle\gamma v_{2}, \gamma v_{2}\right\rangle=\left\langle\xi v_{1}+v_{2}, \xi v_{1}+v_{2}\right\rangle=2 \xi$ so that $\xi=0$, implying $\gamma v_{2}=v_{2}$. as required.

This completes the proof of theorem 1.2.

Remark Note that the construction exhibits $\Gamma$ explicitly as a subgroup of the stabiliser of the lightlike vector $v_{1}$.

Proof of Theorem 1.1 To complete the proof of Theorem 1.1 we proceed as follows. Let $q_{n+2}$ be the form constructed above and consider $\mathrm{O}_{0}\left(q_{n+2} ; \mathbf{Z}\right)$. As noted in the Remark above, the construction yields $\Gamma$ as a subgroup of the stabiliser in $\mathrm{O}_{0}\left(q_{n+2} ; \mathbf{Z}\right)$ of the light-like vector $v_{1}$, however the proof provides no control over whether $\Gamma$ is actually equal to $\operatorname{Stab}\left(v_{1}\right)$. To achieve this, we show that the subgroup $\Gamma$ is separable in $\mathrm{O}_{0}\left(q_{n+2} ; \mathbf{Z}\right)$ and then the theorem follows by a standard separability argument.

To this end, let $C$ be the maximal peripheral subgroup of $\mathrm{O}_{0}\left(q_{n+2} ; \mathbf{Z}\right)$ fixing $v_{1}$, so that $\Gamma<C<\mathrm{O}_{0}\left(q_{k+2} ; \mathbf{Z}\right)$, and $C$ is a crystallographic group. We recall that by Theorem 2.2, $C$ contains a translational subgroup $T^{*}$ which is free abelian of rank $n$, and is the maximal abelian subgroup of $C$. 
We begin by observing that our construction of the group $\Gamma$ began with a translational subgroup which contained translation by 1 in each of the coordinate directions, so that after the two dilation conjugacies which convert rational to integral, we see that for some integer $p>1$, the maximal translational subgroup $T^{*}$ of $\operatorname{Stab}\left(v_{1}\right) \leq \mathrm{O}_{0}\left(q_{n+2} ; \mathbf{Z}\right)$ contains the group $T_{p}$ consisting of translations by $p$ in each of the coordinate directions of the first $n+1$ coordinates.

We claim that $T_{p}$ is separable in $\mathrm{O}_{0}\left(q_{n+2} ; \mathbf{Z}\right)$. The reason is this: Firstly, any element of $\mathrm{O}_{0}\left(q_{n+2} ; \mathbf{Z}\right)$ which lies outside $T^{*}$ can be separated from $T_{p}$ since in fact it can be separated from $T^{*}$ by Lemma 2.7 .

Secondly, we claim that any element of $T^{*}-T_{p}$ may be separated from $T_{p}$. This involves a few cases, which we now sketch.

In the first place, we observe that all the elements of $\gamma \in T^{*}$ have the first $n+1$ entries of its last row being zero, since $\left\langle\gamma(w), v_{1}\right\rangle=\left\langle w, \gamma^{-1}\left(v_{1}\right)\right\rangle=\left\langle w, v_{1}\right\rangle=0$.

Moreover, if we look at the upper left $n \times n$ block of any element of $T_{p}$, this is constructed to be the identity matrix and if the element $\gamma$ we wish to separate does not have this property then we may separate by choosing a random prime $q$ not dividing some such entry and use the restriction of the homomorphism $\mathrm{SL}(n+2, \mathbf{Z}) \rightarrow \mathrm{SL}(n+2, \mathbf{Z} / q \mathbf{Z})$ to the integral subgroup $\mathrm{O}_{0}\left(q_{n+2} ; \mathbf{Z}\right)$.

It follows that it remains to separate an element $\gamma \in T^{*}-T_{p}$ which is the identity matrix in the first $n+1$ columns save for the first $n$ entries in the $n+1$-st row. It is these entries which contribute to the translational nature of the elements of $T_{p}$. However, recall that we have proved that any isometry of $\langle$,$\rangle which is the identity on the upper left n+1 \times n+1$ block must in fact be the identity matrix. It follows that in the matrix $\gamma$ these entries cannot all be divisible by $p$ (else the upper left $n+1 \times n+1$ block is identical with that for some matrix of $T_{p}$ and we deduce that $\gamma \in T_{p}$ ) so that we may use the reduction map $\mathrm{SL}(n+2, \mathbf{Z}) \rightarrow \mathrm{SL}(n+2, \mathbf{Z} / p \mathbf{Z})$ to separate $\gamma$ in this case.

We note that this argument can actually be used to show a little more, namely that for any integer $r$, the subgroups $T_{r p}$ of $T_{p}$ are separable in $\mathrm{O}_{0}\left(q_{n+2} ; \mathbf{Z}\right)$.

The separability of $\Gamma$ may now be deduced. For if we let $T_{\Gamma}$ be the maximal abelian subgroup of $\Gamma$, then $T_{\Gamma} \cap T_{p}$ is a subgroup of finite index in $T_{p}$ and it follows that there is an integer $r$ for which $T_{r p} \leq T_{\Gamma} \cap T_{p} \leq \Gamma$. The separability of $\Gamma$ follows from Lemma 2.6. This completes the proof of theorem 1.1.

Remark The number of cusps for the groups $\mathrm{O}_{0}\left(q_{n+2} ; \mathbf{Z}\right)$ can be greater than one, even for simple examples. For example, the groups $\mathrm{O}_{0}\left(f_{n} ; \mathbf{Z}\right)$ have 1 cusp for $2 \leq n \leq 8$, but $\mathrm{O}_{0}\left(f_{9} ; \mathbf{Z}\right)$ has 2 cusps. This can be seen from [15] and [16] 
which describes these unit groups as groups generated by reflections in certain ideal simplices in $\mathbf{H}^{n}$. The number of cusps being easy to read off from the Coxeter diagrams.

\section{Example}

We finish off by giving an example of the construction as an aid to the proof of Theorem 1.2. We shall take the Hantsche-Wendt manifold, which arises as the 3 -fold cyclic branched cover of the figure-eight knot. Its fundamental group is therefore a Bieberbach group in dimension 3. Representing matrices are provided on pp. 6-7 of [4] which in our notation are:

$$
\Phi^{*}(a)=\left(\begin{array}{cccc}
-1 & 0 & 0 & 1 / 2 \\
0 & -1 & 0 & 1 / 2 \\
0 & 0 & 1 & 1 / 2 \\
0 & 0 & 0 & 1
\end{array}\right) \quad \text { and } \quad \Phi^{*}(b)=\left(\begin{array}{cccc}
1 & 0 & 0 & 1 / 2 \\
0 & -1 & 0 & 0 \\
0 & 0 & -1 & 0 \\
0 & 0 & 0 & 1
\end{array}\right)
$$

We conjugate to clear fractions and form the representation $\Phi$ and hence $\hat{\Phi}$. In this case an invariant form for the finite holonomy is $D=x^{2}+y^{2}+z^{2}$ and when we solve the equations for the group to be an isometry for the form $D \oplus H_{2}$ we obtain:

$$
\hat{\Phi}(a)=\left(\begin{array}{ccccc}
-1 & 0 & 0 & 0 & 1 \\
0 & -1 & 0 & 0 & 1 \\
0 & 0 & 1 & 0 & 1 \\
1 & 1 & -1 & 1 & -\frac{3}{2} \\
0 & 0 & 0 & 0 & 1
\end{array}\right) \quad \text { and } \hat{\Phi}(b)=\left(\begin{array}{ccccc}
1 & 0 & 0 & 0 & 1 \\
0 & -1 & 0 & 0 & 0 \\
0 & 0 & -1 & 0 & 0 \\
-1 & 0 & 0 & 1 & -\frac{1}{2} \\
0 & 0 & 0 & 0 & 1
\end{array}\right)
$$

Letting $K=2$, and conjugating, gives integral matrices,

$$
\hat{\Phi}(a)=\left(\begin{array}{ccccc}
-1 & 0 & 0 & 0 & 2 \\
0 & -1 & 0 & 0 & 2 \\
0 & 0 & 1 & 0 & 2 \\
1 & 1 & -1 & 1 & -3 \\
0 & 0 & 0 & 0 & 1
\end{array}\right) \quad \text { and } \quad \hat{\Phi}(b)=\left(\begin{array}{ccccc}
1 & 0 & 0 & 0 & 2 \\
0 & -1 & 0 & 0 & 0 \\
0 & 0 & -1 & 0 & 0 \\
-1 & 0 & 0 & 1 & -1 \\
0 & 0 & 0 & 0 & 1
\end{array}\right)
$$

preserving the rationally equivalent form $x^{2}+y^{2}+z^{2}+4 w t$.

Note that the above form is equivalent over $\mathbf{Q}$ to $f_{4}=x^{2}+y^{2}+z^{2}+w^{2}-t^{2}$. In [13] the authors obtain the Hantsche-Wendt manifold as a cusp cross-section of a hyperbolic 4-manifold arising from a torsion-free subgroup in $O_{0}\left(f_{4} ; \mathbf{Z}\right)$.

Acknowledgements The first author was partially supported by the N.S.F, and the second was partially supported by the N.S.F, the Alfred P. Sloan Foundation and a grant from the Texas Advanced Research Program. 


\section{References}

[1] I. Agol, D.D. Long and A.W. Reid, The Bianchi groups are separable on geometrically finite subgroups, Annals of Math. 153 (2001), pp. 599-621.

[2] A. Borel, Compact Clifford-Klein forms of symmetric spaces, Topology 2 (1963), pp. $111-122$.

[3] A. Borel and Harish-Chandra, Arithmetic subgroups of algebraic groups, Annals of Math. 75 (1962), 485-535.

[4] L.S. Charlap, Bieberbach Groups and Flat Manifolds, Universitext, SpringerVerlag (1986).

[5] F.T. Farrell and S. Zdravkovska, Do almost flat manifolds bound, Michigan J. Math. 30 (1983), pp 199-208.

[6] M.L. Gromov, Almost flat manifolds, J. Diff. Geom. 13 (1978), pp 231-241.

[7] G. Hamrick and D. Royster, Flat Riemannian manifolds are boundaries, Invent. Math. 66 (1982), pp. 405 -413.

[8] T.Y. Lam, The Algebraic Theory of Quadratic Forms, Benjamin (1973).

[9] D.D. Long, Immersions and embeddings of totally geodesic surfaces, Bull. London Math. Soc. 19 (1987), pp. 481-484.

[10] D.D. Long and A.W. Reid, On the geometric boundaries of hyperbolic 4manifolds, Geometry and Topology, 4 (2000), pp. 171-178.

[11] B.E. Nimershiem, All flat three-manifolds appear as cusps of hyperbolic fourmanifolds, Topology and Its Appl. 90 (1998), pp. 109-133.

[12] J.G. Ratcliffe, Foundations of Hyperbolic Manifolds, G.T.M. 149, SpringerVerlag, (1994).

[13] J.G. Ratcliffe and S.T. Tschantz, The volume spectrum of hyperbolic 4manifolds, Experimental Math 9 (2000), pp. 101-125.

[14] W.P. Thurston, Three-Dimensional Geometry and Topology, Volume 1, Princeton University Press (1997).

[15] E.B. Vinberg, On groups of unit elements of certain quadratic forms, Mat. Sb. 87 (1972) pp. 17-35.

[16] E.B. Vinberg and O.V. Shvartsman, Discrete groups of motions of spaces of constant curvature, Geometry II, Encyc. Math. Sci. 29, pp 139-248, SpringerVerlag (1993).

Department of Mathematics, University of California

Santa Barbara, CA 93106, USA

and

Department of Mathematics, University of Texas

Austin, TX 78712, USA

Email: long@math.ucsb.edu, areid@math.utexas.edu

Received: 6 December 2001

Algebraic 63 Geometric Topology, Volume 2 (2002) 